\title{
Approximate Solutions to the Klein-Fock-Gordon Equation for the Sum of Coulomb and Ring-Shaped-Like Potentials
}

\author{
Sh. M. Nagiyev, ${ }^{1}$ A. I. Ahmadov $\mathbb{D}^{2,3}$ and V. A. Tarverdiyeva ${ }^{1}$ \\ ${ }^{1}$ Institute of Physics, Azerbaijan National Academy of Sciences, H. Javid Avenue, 131, AZ-1143 Baku, Azerbaijan \\ ${ }^{2}$ Department of Theoretical Physics, Baku State University, Z. Khalilov St. 23, AZ-1148 Baku, Azerbaijan \\ ${ }^{3}$ Institute of Physical Problems, Baku State University, Z. Khalilov St. 23, AZ-1148 Baku, Azerbaijan
}

Correspondence should be addressed to A. I. Ahmadov; ahmadovazar@yahoo.com

Received 6 March 2020; Accepted 20 April 2020; Published 11 June 2020

Academic Editor: Shi-Hai Dong

Copyright ( 2020 Sh. M. Nagiyev et al. This is an open access article distributed under the Creative Commons Attribution License, which permits unrestricted use, distribution, and reproduction in any medium, provided the original work is properly cited. The publication of this article was funded by $\mathrm{SCOAP}^{3}$.

We consider the quantum mechanical problem of the motion of a spinless charged relativistic particle with mass $M$, described by the Klein-Fock-Gordon equation with equal scalar $S(\vec{r})$ and vector $V(\vec{r})$ Coulomb plus ring-shaped potentials. It is shown that the system under consideration has both a discrete at $|E|<M c^{2}$ and a continuous at $|E|>M c^{2}$ energy spectra. We find the analytical expressions for the corresponding complete wave functions. A dynamical symmetry group $S U(1,1)$ for the radial wave equation of motion is constructed. The algebra of generators of this group makes it possible to find energy spectra in a purely algebraic way. It is also shown that relativistic expressions for wave functions, energy spectra, and group generators in the limit $c \longrightarrow \infty$ go over into the corresponding expressions for the nonrelativistic problem.

\section{Introduction}

Nonrelativistic Schrödinger and relativistic Dirac, KleinFock-Gordon (KFG), and finite-difference equations describe the systems in the nuclear physics, elementary particle physics, and atomic and molecular physics [1-6]. Examples to the commonly used potentials in these equations are the Coulomb potential and the harmonic oscillator potential, as well as their various varieties. Other types of interaction potentials included were the Kratzer [7], Morse [8], Eckart [9], Manning-Rosen [10], Pöschl-Teller [11], Hulthèn [12], Wood-Saxon [13], Makarov [14], Hartmann [15], and Hautot [16] potentials. Exact solvability and the application range of these potentials are examples to the main properties of them. Several approaches were developed to study the quantum systems either relativistic or nonrelativistic with nonzero angular momentum [17-23].

The numerous interesting works devoted to the study of the KFG equation with different potentials. The s-wave
KFG equation with the vector Hulthèn-type potential is one of the examples which was investigated by the use of the standard methods. Afterwards, the further study for the vector and scalar Hulthèn-type potentials was carried out by Dominguez-Adame and Chen et al. [24, 25]. The same potential for the s-wave KFG equation was obtained for regular and irregular boundary conditions in Ref. [26]. The path integral approach to the Green function for the KFG equation with these two potentials was studied in Ref. [27]. It should also be noted that the KFG equation has been recently studied with various combined potentials, for example, Hulthén plus ring-shaped potentials [28] and Manning-Rosen plus a Class of Yukawa potentials [29] by using ordinary and SUSY quantum mechanics.

In Ref. [30-35], the scalar potential is equal and not equal to the vector potential which was assumed to get the bound states of the KFG equation with some typical potential by using the ordinary quantum mechanics. Furthermore, the KFG equation with the ring-shaped-like potential was 
investigated by Dong and Lozada-Cassou [34]. If we consider the case where the interaction potential is not enough to create particle-antiparticle pairs, the KFG equation can be applied to the treatment of a zero-spin particle and apply the Dirac equation to that of a $1 / 2$-spin particle. When a particle is in a strong field, the relativistic wave equations should be considered in the quantum system. In any case, we can make the correction easily for nonrelativistic quantum mechanics.

Since the Coulomb potential is one of the exactly solvable potentials in physics, it has a wide range of application to the several research areas such as nuclear and particle, atomic, condensed matter, and chemical physics. It is a natural and interesting question to study the relativistic effects for a particle within this potential especially for strong coupling. Notice that the KFG equation cannot be solved exactly for many potentials of interactions for nonzero angular momentum $l \neq 0$-states because of the centrifugal term of potentials.

Noncentral potentials which play an important role for describing the quantum systems have the following form in spherical coordinates:

$$
V(r, \theta, \phi)=V(r)+\frac{f(\theta, \phi)}{r^{2}}
$$

where $V(r)$ and $f(\theta, \phi)$ are certain functions of their arguments. Coulomb or harmonic oscillator potentials are frequently used for the central part of equation (1).

One of the convenient combinations is the Hartmann potential [15] for which

$$
V=\eta \sigma^{2}\left(\frac{2 a_{0}}{r}-\eta \frac{a_{0}^{2}}{r^{2} \sin ^{2} \theta}\right) \varepsilon_{0}
$$

here, $a_{0}$ is the Bohr radius, $a_{0}=\hbar^{2} / m e^{2}, \varepsilon_{0}$ is the ground state energy of the hydrogen atom, $\varepsilon_{0}==m e^{4} / 2 \hbar^{2}$, and $\eta$ and $\sigma$ are positive real numbers. This potential was proposed by Hartmann in the framework of the nonrelativistic quantum mechanics for describing the organic molecules such as benzene within quantum chemistry. One can use the same potential in nuclear physics for studying the interactions between deformed nuclei.

Other significant noncentral potentials of the form (1) were considered by Hautot [16] in the framework of the nonrelativistic quantum mechanics to study the problem of the motion of a charged particle. He considered the two- and three-dimensional harmonic oscillator potentials and the Coulomb potential to which terms of the type $f(\theta) / r^{2}$ were added and found such functions $f(\theta)$ that enabled him to solve exactly the corresponding Schrödinger equations. The original Hautot potential is defined in the following form:

$$
V(r, \theta)=\frac{\mu}{e}\left[V(r)+\frac{f(\theta)}{r^{2}}\right],
$$

here, $\mu$ is the mass of the charged particle and $e$ is the charge of the particle.
Hence, the Hartmann potential is a special case of the Hautot potentials.

One needs to study the noncentral potentials rather than the central ones for getting better results in molecular structures and interactions. Examples to these situations include the use of the ring-shaped potentials in quantum chemistry to describe the ring-shaped organic molecules and in nuclear physics to investigate the interaction between the deformed nucleus and the spin-orbit coupling for a motion of the particle in the potential fields. This noncentral potential is also used as a mathematical model in the description of diatomic molecular vibrations, and it constitutes a convenient model for other physical situations.

Coulomb plus ring-shaped potential was studied for the three-dimensional motion of a charged relativistic quantum particle in a noncentral potential [36]. This investigation is based on a finite-difference version of relativistic quantum mechanics (see references in [5]) and is a generalization of the results of [16] to the relativistic case. We emphasize that the Coulomb plus ring-shaped-like potentials (3) are widely used in various fields of physics: in nuclear and particle physics, in atomic and molecular physics, and in condensed matter and chemical physics in the nonrelativistic, as well as in the relativistic regions.

Possible application of the combined potentials can be the study of the interaction between deformed pair of the nucleus and spin-orbit coupling in nuclear physics. Another possibility in the hadronic systems may be the description of vibrations which can shed light on other physical situations. In conclusion, the study of the wave functions and the energies in the bound and continuum states of the interacting systems with the linear combination of Coulomb and ring-shaped potentials is quite impressive, and it can provide a deeper and genuine appreciation of the physical properties.

The remainder of the present work proceeds in the following order: in Section 2, we give the information about the relativistic model of the Coulomb plus ring-shaped-like potential. Next, we present the solutions of the radial KFG equation in Section 3. Then, in Section 4, we give the dynamical group of symmetry of the present system. In Section 5, we present the solution of the angle-dependent part of the KFG equation. In Section 6, we obtain the nonrelativistic limit of the eigenfunctions and the energy spectrum, and some concluding remarks are stated in Section 7.

\section{The Relativistic Model of the Coulomb plus Ring-Shaped-Like Potential}

The KFG equation with $S(\mathbf{r})$ scalar and $V(\mathbf{r})$ vector potentials has the form [1]

$$
\left\{-\hbar^{2} c^{2} \nabla^{2}+\left[M c^{2}+S(\mathbf{r})\right]^{2}-[E-V(\mathbf{r})]^{2}\right\} \psi(\mathbf{r})=0
$$

In the nonrelativistic limit, this equation transforms into the Schrödinger equation for the sum of potentials $V_{N}$ and 
$S_{N}$, i.e.,

$$
\left[-\frac{\hbar^{2}}{2 M} \Delta+V_{N}(\mathbf{r})+S_{N}(\mathbf{r})-E_{N}\right] \psi_{N}(\mathbf{r})=0
$$

where $E_{N}=\lim _{c \rightarrow \infty}\left(E-M c^{2}\right), V_{N}(\mathbf{r})=\lim _{c \rightarrow \infty} V(\mathbf{r})$, and $S_{N}(\mathbf{r})=$ $\lim S(\mathbf{r})$.

We present here the exact solutions of the KFG equation with equal scalar and vector potentials for the sum Coulomb and ring-shaped potentials of the type (1). We also use an approach based on the Lie algebra of the group $S U(1,1)$, well known to be the dynamical group for several quantum systems [37-40]. We introduce a tilting transformation that relates between the physical states and the group sates which constitute a basis of the relevant unitary irreducible representation of $S U(1,1)$.

As done in the literatures [30-35], here, we assume that

$$
S(r)=V(r)=-\frac{\alpha_{E}}{r}+\frac{f(\theta, \phi)}{r^{2}} .
$$

In terms of dimensionless variables $\boldsymbol{\rho}=\mathbf{r} / \lambda, f_{0}=M$ $f(\theta, \phi) / \hbar^{2}$ and parameters $\alpha_{0}=\alpha_{E} / \hbar c, \varepsilon=E / M c^{2}$, equation (4) takes the form

$$
H \psi \equiv\left[-\nabla_{\rho}^{2}+2(1+\varepsilon)\left(-\frac{\alpha_{0}}{\rho}+\frac{f_{0}}{\rho^{2}}\right)+1-\varepsilon^{2}\right] \psi(\rho, \theta, \phi)=0 .
$$

Here, the operator $\nabla_{\rho}^{2}$ has the usual form

$$
\begin{aligned}
\nabla_{\boldsymbol{\rho}}^{2} & =\partial_{\rho}^{2}+\frac{2}{\rho} \partial_{\rho}-\frac{\widehat{\mathbf{L}}^{2}}{\rho^{2}}, \\
\partial_{\rho} & =\frac{\partial}{\partial \rho}, \\
\boldsymbol{\rho} & =(\rho, \theta, \phi),
\end{aligned}
$$

where $\widehat{\mathbf{L}}^{2}$ is the square of the angular momentum operator, and it is defined as

$$
\widehat{\mathbf{L}}^{2}=-\Delta_{\theta, \phi}=-\left[\frac{1}{\sin \theta} \partial_{\theta}\left(\sin \theta \partial_{\theta}\right)+\frac{1}{\sin ^{2} \theta} \partial_{\phi}^{2}\right]
$$

Equation (7) does not differ in form from the Schrödinger equation (5). It allows separation of variables in the spherical coordinates $\rho, \theta, \phi(0 \leq \rho<\infty, 0 \leq \theta \leq \pi, 0 \leq \phi<2 \pi)$.

Searching the wave function in the form

$$
\psi(\rho, \theta, \phi)=\rho^{-1} R(\rho) F(\theta, \phi)
$$

one obtains the set of separated differential equations

$$
\left(\partial_{\rho}^{2}+\frac{2(1+\varepsilon) \alpha_{0}}{\rho}-\frac{g}{\rho^{2}}-1+\varepsilon^{2}\right) R=0
$$

$$
(\widehat{A}-g) F(\theta, \phi)=0
$$

here, $g$ is a separation constant and

$$
\begin{aligned}
\widehat{A} & =-\Delta_{\theta, \phi}+M_{0} f(\theta, \phi), \\
M_{0} & =\frac{2 M(1+\varepsilon)}{\hbar^{2}} .
\end{aligned}
$$

The operator $\widehat{A}$ depends on energy, so its eigenvalues $g$ will also depend on energy, $g=g(\varepsilon)$. These dependences lead to the fact that an equation determining the energy levels (see formula (67)) will be very complicated. However, in the nonrelativistic limit, these dependences disappear, i.e.,

$$
\begin{aligned}
\lim _{c \rightarrow \infty} \widehat{A} & =\widehat{A}_{N}=-\Delta_{\theta, \phi}+\frac{4 M f_{N}(\theta, \phi)}{\hbar^{2}}, \\
\lim _{c \rightarrow \infty} g(\varepsilon) & =g_{N} .
\end{aligned}
$$

The operator $\widehat{A}$ commute with the Hamiltonian $H$, i.e., [ $H, \widehat{A}]=0$. It is responsible for separability of $H$ in the spherical coordinates.

\section{The Solutions of the Radial Klein-Fock- Gordon Equation}

Let us go to the radial wave equation (11). Here, we suppose that the $\varepsilon$ and $g$ are arbitrary parameters. Putting

$$
R(\rho)=\rho^{v} e^{-\sqrt{1-\varepsilon^{2}} \rho} \Omega(\rho), \quad v=\frac{1}{2}+\sqrt{\frac{1}{4}+g}
$$

in (11), there we receive the following equation for the function $\Omega(\rho)$ :

$\rho \Omega^{\prime \prime}+2\left(\nu-\sqrt{1-\varepsilon^{2}} \rho\right) \Omega^{\prime}+\left(2(1+\varepsilon) \alpha_{0}-2 \sqrt{1-\varepsilon^{2}} v\right) \Omega=0$.

If we compare this equation with the following equation

$$
z u^{\prime \prime}+(\gamma-z) u^{\prime}-\alpha u=0
$$

for a confluent hypergeometric function, its solutions are expressed in the form:

$$
\Omega(\rho)=F\left(v-\frac{(1+\varepsilon) \alpha_{0}}{\sqrt{1-\varepsilon^{2}}}, 2 v ; 2 \sqrt{1-\varepsilon^{2}} \rho\right) .
$$

Consequently,

$$
R(\rho)=\rho^{v} e^{-\sqrt{1-\varepsilon^{2}} \rho} F\left(v-\sqrt{\frac{\varepsilon+1}{\varepsilon-1}} \alpha_{0}, 2 v ; 2 \sqrt{1-\varepsilon^{2}} \rho\right) .
$$

To analyze the obtained solutions, we consider separately the cases when $|\varepsilon|<1$ (or $|E|<M c^{2}$ ) and $|\varepsilon|>1$ (or $|E|>M c^{2}$ ). 
(1) Let be $|\varepsilon|<1$. From the condition that the wave function is finite at zero $R(0)=0$ follows that $v>0$. This case corresponds to the discrete energy spectrum of our system. Let us find the energy spectrum and wave function. Demand $R(\infty)=0$ for the wave function (19) leads to the following condition for energy quantization:

$$
v-\sqrt{\frac{\varepsilon+1}{\varepsilon-1}} \alpha_{0}=-n, \quad n=0,1,2, \cdots
$$

where $n$ denotes the radial quantum number.

It follows from (20) the discrete energy level equation for our system in the case of $0<|\varepsilon|<1$

$$
(n+v) \sqrt{1-\varepsilon}=\alpha_{0} \sqrt{1+\varepsilon}, \quad n=0,1,2, \cdots
$$

where the parameter $v$ may only take special values to be determined from (12). We emphasize that for hydrogenlike atoms, $\alpha_{0}$ must be replaced by $Z \alpha_{0}$. Then for the energies $-1<\varepsilon<0$, equation (21) will be satisfied for sufficiently large values $Z$. For example, when $n=0, v=1$, we get $Z=\left(\nu / \alpha_{0}\right)$ $\sqrt{(1+|\varepsilon|) /(1-|\varepsilon|)}$. From here when $|\varepsilon|=0.2$, we find $Z \geq 167$

Thus, it follows from (19) and (20) that the radial wave functions corresponding to discrete energy levels (21) will have the form

$$
R_{n}(\rho)=\tilde{C}_{n} \rho^{v} e^{-\sqrt{1-\varepsilon_{n}^{2}} \rho} F\left(-n, 2 v ; 2 \sqrt{1-\varepsilon_{n}^{2} \rho}\right)
$$

here, $\varepsilon_{n}$ are roots of equation (21). Using formulas [41]:

$$
L_{n}^{\alpha}(x)=\frac{(\alpha+1)_{n}}{n !} F(-n, \alpha+1 ; x)
$$

the functions $R_{n}(\rho)$ can be expressed by the associated Laguerre polynomials. As a result, we conclude that the normalized radial wave functions, corresponding to the discrete energy spectrum (21), are

$$
R_{n}(\rho)=C_{n} \rho^{v} e^{-\sqrt{1-\varepsilon_{n}^{2}} \rho} L_{n}^{2 v-1}\left(2 \sqrt{1-\varepsilon_{n}^{2}} \rho\right),
$$

where $C_{n}$ is the normalization constant

$$
C_{n}=2^{v} \sqrt{\frac{n !\left(1-\varepsilon_{n}^{2}\right)^{v+1 / 2}}{2(n+v) \Gamma(n+2 v)}} .
$$

It is found from the following condition

$$
\int_{0}^{\infty} R_{n}^{2}(\rho) d \rho=1
$$

To calculate this integral, the standard trick was used, i.e., recurrence relation

$x L_{n}^{\alpha}(x)=(2 n+\alpha+1) L_{n}^{\alpha}(x)-(n+1) L_{n+1}^{\alpha}(x)-(n+1) L_{n-1}^{\alpha}(x)$

and orthogonality properties for associated Laguerre polynomials:

$$
\int_{0}^{\infty} x^{\alpha} e^{-x} L_{n}^{\alpha}(x) L_{m}^{\alpha} d x=\frac{\Gamma(n+\alpha+1)}{n !} \delta_{n m} .
$$

(2) Let now $|\varepsilon|>1$. In this case, the energy spectrum will be continuous, and the corresponding wave functions are obtained from expression (19). For example, for $\varepsilon>1$ (in this case $v$ is a real parameter)

$$
\begin{array}{r}
R(\rho)=C \rho^{v} e^{-i \tau \rho} F\left(\nu+\frac{i(1+\varepsilon) \alpha_{0}}{\sqrt{\varepsilon^{2}-1}}, 2 v ; 2 i \sqrt{\varepsilon^{2}-1} \cdot \rho\right), \\
\tau=\sqrt{\varepsilon^{2}-1} .
\end{array}
$$

Radial wave functions corresponding to energy values $\varepsilon=0, \pm 1$, can be obtained from (19) by using the corresponding passage to the limit. For example, for the value $\varepsilon=1$, we find

$$
\lim _{\varepsilon \rightarrow 1} R(\rho)=C^{\prime} \rho^{-v_{1}+1} J_{2 v_{1}-1}\left(4 \sqrt{\alpha_{0} \rho}\right)
$$

where $v_{1}=\lim _{\varepsilon \rightarrow 1} v, J_{v}(z)$ are well known Bessel functions. In the derivation of (30), we used the easily proved limit formula

$$
\lim _{\alpha \rightarrow 0} F\left(\nu-\frac{a}{\alpha}, \gamma ; \alpha x\right)=\Gamma(\gamma+1)(a x)^{-\gamma+1} J_{\gamma-1}(2 \sqrt{a x}) .
$$

The asymptotic behavior of Bessel functions at zero and at infinity is given by the formulas, respectively [41].

$$
\begin{aligned}
& \underset{\substack{v \\
z \rightarrow 0}}{J_{2}(z)} \approx\left(\frac{z}{2}\right)^{v}, \\
& \underset{\substack{v \\
z \rightarrow \infty}}{J_{v}(z)} \approx \sqrt{\frac{2}{\pi z}}\left[\cos \left(z-\frac{v \pi}{2}-\frac{\pi}{4}\right)-\frac{1}{z}\left(v^{2}-\frac{1}{4}\right) \sin \left(z-\frac{v \pi}{4}-\frac{\pi}{4}\right)\right] .
\end{aligned}
$$

It follows that the wave function (31) is nonquadratic integrable at zero and at infinity. This means that the radial equation (11) does not have quadratically integrable solutions; therefore, a charged particle (e.g., electron) in the Coulomb plus ring-shaped potential field (6) of an arbitrary charge $\alpha_{E}=Z e^{2}$ does not have bound states with energy $E=M c^{2}$, i.e., with zero binding energy. The nature of this phenomenon, as in the case of the Dirac equation considered in [42], is associated with the long-range nature of the Coulomb potential. The fact is that this potential 
generates an infinite set of bound states with energy equations defined by formula (21). This set accumulates at a point $E=M c^{2}$ but does not reach this point. Such a picture remains valid for any value of the charge $Z$. This conclusion can also be obtained based on the wave function (29).

\section{Dynamical Symmetry Group}

Let us now consider the radial equation (11) by the help of $S U(1,1)$ Lie algebra. The generators of $S U(1,1)$ algebra may be realized as [37]

$$
\begin{aligned}
& K_{0} \equiv \Gamma_{0}=\frac{1}{2}\left(-\rho \partial_{\rho}^{2}+\frac{g}{\rho}+\rho\right), \\
& K_{1} \equiv \Gamma_{4}=\frac{1}{2}\left(-\rho \partial_{\rho}^{2}+\frac{g}{\rho}-\rho\right), \\
& K_{2} \equiv T=-i \rho \partial_{\rho} .
\end{aligned}
$$

By a direct check, one can verify that these operators satisfy the commutation relations

$$
\begin{aligned}
{\left[\Gamma_{0}, \Gamma_{4}\right] } & =i T, \\
{\left[T, \Gamma_{0}\right] } & =i \Gamma_{4}, \\
{\left[\Gamma_{4}, T\right] } & =-i \Gamma_{0} .
\end{aligned}
$$

The Casimir operator [38] is

$$
C_{2}=\Gamma_{0}^{2}-\Gamma_{4}^{2}-T^{2}=s(s-1)
$$

We denote the states of a positive discrete series as $|n, s\rangle$ such that

$$
\begin{aligned}
& \Gamma_{0}|n, s>=(n+s)| n, s>, \\
& C_{2}|n, s>=s(s-1)| n, s>,
\end{aligned}
$$

where $s$ is the Bargmann index, $s>0$, and $n=0,1,2, \cdots$. It should be noted that in our case from equation (33), we obtain $C_{2}=g=v(v-1)$, so $s=v$. Equation (11) can be written with the help of generators (33) as

$$
\left[\left(2-\varepsilon^{2}\right) \Gamma_{0}+\varepsilon^{2} \Gamma_{4}-2(1+\varepsilon) \alpha_{0}\right] R=0
$$

Let us introduce a supplementary parameter $\theta$ and perform a tilting transformation

$$
\tilde{R}=S R, \quad S=e^{-i \theta T}
$$

From the commutation relations (34) and the formula

$$
e^{\widehat{A}} \widehat{B} e^{-\widehat{A}}=\widehat{B}+[\widehat{A}, \widehat{B}]+\frac{1}{2 !}[\widehat{A},[\widehat{A}, \widehat{B}]]+\cdots
$$

where $\widehat{A}$ and $\widehat{B}$ are any two operators, it follows that

$$
\begin{aligned}
e^{-i \theta T} \Gamma_{0} e^{i \theta T} & =\Gamma_{0} \cosh \theta+\Gamma_{4} \sinh \theta, \\
e^{-i \beta \Gamma_{0}} \Gamma_{4} e^{i \beta \Gamma_{0}} & =\Gamma_{4} \cos \beta+T \sin \beta, \\
e^{-i \theta T} \Gamma_{4} e^{i \theta T} & =\Gamma_{4} \cosh \theta+\Gamma_{0} \sinh \theta, \\
e^{-i \alpha \Gamma_{4}} \Gamma_{0} e^{i \alpha \Gamma_{4}} & =\Gamma_{0} \cosh \alpha-T \sinh \alpha, \\
e^{-i \beta \Gamma_{0}} T e^{i \beta \Gamma_{0}} & =T \cos \beta-\Gamma_{4} \sin \beta, \\
e^{-i \alpha \Gamma_{4}} T e^{i \alpha \Gamma_{4}} & =T \cosh \alpha-\Gamma_{0} \sinh \alpha .
\end{aligned}
$$

Through using formula (40), it is easy to verify that equation (37) becomes

$$
\begin{aligned}
& {\left[\left(\left(2-\varepsilon^{2}\right) \cosh \theta+\varepsilon^{2} \sinh \theta\right) \Gamma_{0}+\left(\left(2-\varepsilon^{2}\right) \sinh \theta\right.\right.} \\
& \left.\left.\quad+\varepsilon^{2} \cosh \theta\right) \Gamma_{4}-2(1+\varepsilon) \alpha_{0}\right] \tilde{R}=0 .
\end{aligned}
$$

To solve equation (41) in an algebraic way, we consider separately the cases when $|\varepsilon|<1$ (or $|E|<M c^{2}$ ) and $|\varepsilon|>1$ (or $|E|>M c^{2}$ ).

(1) When $|\varepsilon|<1$ (discrete spectrum) in (41), a compact generator $\Gamma_{0}$ can be diagonalized. Setting the coefficient of $\Gamma_{4}$ to zero, we obtain $\tanh \theta=-\varepsilon^{2} /\left(2-\varepsilon^{2}\right)$ or $e^{\theta}=\sqrt{1-\varepsilon^{2}}$. As a result, we have

$$
\left(\sqrt{1-\varepsilon^{2}} \Gamma_{0}-(1+\varepsilon) \alpha_{0}\right) \tilde{R}=0
$$

It follows from (42) the energy level equation (21).

(2) When $|\varepsilon|>1$, noncompact generator $\Gamma_{4}$ is diagonalized, having a continuous real spectrum $\lambda \in R$. In this case, we equate to zero the coefficient of the operator $\Gamma_{0}$, i.e., $\left(2-\varepsilon^{2}\right) \cosh \theta+\varepsilon^{2} \sinh \theta=0$. From here, we get $\tanh \theta=-\left(2-\varepsilon^{2}\right) / \varepsilon^{2}$ and $e^{\theta}=\sqrt{-\left(1-\varepsilon^{2}\right)}$. Moreover, equation (41) takes the form

$$
\left(\sqrt{-\left(1-\varepsilon^{2}\right)} \Gamma_{4}-(1+\varepsilon) \alpha_{0}\right) \tilde{R}_{1}=0
$$

Consequently,

$$
E_{\lambda}=\frac{\lambda^{2}+\alpha_{0}^{2}}{\lambda^{2}-\alpha_{0}^{2}} M c^{2} .
$$

We emphasize that the generators $\Gamma_{0}, \Gamma_{4}$, and $T$ have no nonrelativistic limit: they diverge at $c \longrightarrow \infty$. Generators $\Gamma_{0}^{\prime}, \Gamma_{4}^{\prime}$, and $T^{\prime}$, which have the correct nonrelativistic limit, can be obtained from $\Gamma_{0}, \Gamma_{4}$, and $T$ by the help of unitary transformation (compare with [43]). Unitary operator $U$, 
performing such a transformation, has the form

$$
\begin{aligned}
U & =e^{-i \omega T}, \\
\tanh \omega & =\frac{1-\varepsilon^{2}-\alpha_{0}^{2}}{1-\varepsilon^{2}+\alpha_{0}^{2}}, \\
e^{\omega} & =\frac{\sqrt{1-\varepsilon^{2}}}{\alpha_{0}} .
\end{aligned}
$$

Then, we will have

$$
\begin{aligned}
& \Gamma_{0}^{\prime}=U \Gamma_{0} U^{-1}=\Gamma_{0} \cosh \omega+\Gamma_{4} \sinh \omega=\frac{1}{2}\left(-\xi \partial_{\xi}^{2}+\frac{g}{\xi}+\xi\right), \\
& \Gamma_{4}^{\prime}=U \Gamma_{4} U^{-1}=\Gamma_{4} \cosh \omega+\Gamma_{0} \sinh \omega=\frac{1}{2}\left(-\xi \partial_{\xi}^{2}+\frac{g}{\xi}-\xi\right), \\
& T^{\prime}=U T U^{-1}=T=-i \xi \partial_{\xi} .
\end{aligned}
$$

In the formulas (46), the following dimensionless variable is introduced $\xi=r / a_{0}$, where $a_{0}=\hbar^{2} / M \alpha_{E}$ is a Bohr radius. It is associated with $\rho=r / \lambda$ in the following way $\xi=\alpha_{0} \rho$. We can also write relations (46) in matrix form

$$
\left(\begin{array}{c}
\Gamma_{0}^{\prime} \\
\Gamma_{4}^{\prime} \\
T^{\prime}
\end{array}\right)=\left(\begin{array}{ccc}
\cosh \omega & \sinh \omega & 0 \\
\sinh \omega & \cosh \omega & 0 \\
0 & 0 & 1
\end{array}\right)\left(\begin{array}{c}
\Gamma_{0} \\
\Gamma_{4} \\
T
\end{array}\right)
$$

As known, under unitary transformations, the Casimir operator remains unchanged, i.e., $C_{2}^{\prime}=U C_{2} U^{-1}=C_{2}=g$.

Equation (11) can also be solved algebraically using the generators $\Gamma_{0}^{\prime}, \Gamma_{4}^{\prime}$, and $T^{\prime}$. To do this, we rewrite it in the form

$$
\begin{aligned}
\left(\alpha_{1} \Gamma_{0}^{\prime}+\alpha_{2} \Gamma_{4}^{\prime}-2(1+\varepsilon) \alpha_{0}\right) R & =0 \\
\alpha_{1,2} & =\frac{\alpha_{0}^{2} \pm\left(1-\varepsilon^{2}\right)}{\alpha_{0}} .
\end{aligned}
$$

We now perform the tilting transformation to remove the noncompact generator $\Gamma_{4}$ [37-40]. To this end, we define

$$
\tilde{R}^{\prime}=S^{\prime} R, \quad S^{\prime}=e^{-i \theta^{\prime} T} .
$$

In view of formulas (40), we rewrite equation (48) in the form

$$
\begin{aligned}
& {\left[\left(\alpha_{1} \cosh \theta^{\prime}+\alpha_{2} \sinh \theta^{\prime}\right) \Gamma_{0}^{\prime}+\left(\alpha_{1} \sinh \theta^{\prime}+\alpha_{2} \cosh \theta^{\prime}\right) \Gamma_{4}^{\prime}\right.} \\
& \left.\quad-2(1+\varepsilon) \alpha_{0}\right] \tilde{R}^{\prime}=0 .
\end{aligned}
$$

If we choose now $\theta^{\prime}$ as

$$
\begin{aligned}
\tanh \theta^{\prime} & =-\frac{\alpha_{2}}{\alpha_{1}}=\frac{1-\varepsilon^{2}-\alpha_{0}^{2}}{1-\varepsilon^{2}+\alpha_{0}^{2}}, \\
e^{\theta^{\prime}} & =\frac{\sqrt{1-\varepsilon^{2}}}{\alpha_{0}},
\end{aligned}
$$

which corresponds to the case $0<|\varepsilon|<1$; then, in (50), the generator $\Gamma_{0}^{\prime}$ is diagonalized, i.e.,

$$
\left(\sqrt{1-\varepsilon^{2}} \Gamma_{0}^{\prime}-(1+\varepsilon) \alpha_{0}\right) \tilde{R}^{\prime}=0
$$

We obtain from (52) again the discrete energy spectrum (21) pure algebraically.

Now we give the relationship between the functions $\tilde{R}$ and $\tilde{R}^{\prime}$. It has the form

$$
\tilde{R}^{\prime}=\tilde{S} \tilde{R}, \quad \tilde{S}=S\left(S^{\prime}\right)^{-1}=e^{-i \tilde{\theta} T}, \tilde{\theta}=\theta-\theta^{\prime}
$$

moreover, $\tanh \tilde{\theta}=\left(\alpha_{0}^{2}-1\right) /\left(\alpha_{0}^{2}+1\right)$ and $e^{\tilde{\theta}}=\alpha_{0}$. Consequently,

$$
\tilde{R}_{n}^{\prime}(\xi)=e^{-i \tilde{\theta} T} \tilde{R}_{n}(\rho)=\tilde{R}_{n}\left(\alpha_{0} \rho\right)
$$

When $|\varepsilon|>1$, we can diagonalized generator $\Gamma_{4}^{\prime}$, and we get the equation

$$
\left(\sqrt{\varepsilon^{2}-1} \Gamma_{4}^{\prime}-(1+\varepsilon) \alpha_{0}\right) \tilde{R}^{\prime}=0
$$

From here follows a continuous energy spectrum (44).

\section{The Solutions of the Angular Equation}

We now investigate the solutions of the angle-dependent equation (12). For this aim, we choose a function $f(\theta, \phi)$ in the form

$$
f(\theta, \phi)=\frac{\gamma+\beta \cos \theta+\alpha \cos ^{2} \theta}{\sin ^{2} \theta} .
$$

In this case, $z$-projection of the angular momentum operator $\widehat{L}_{z}=-i \partial_{\phi}$ will also commute with the Hamiltonian $H$. Thus, the variables in equation (12) can be further separated in the usual way

$$
F(\theta, \phi)=\frac{\Theta(\theta) e^{i m \phi}}{\sqrt{2 \pi}} .
$$

Here, $m$ is the usual magnetic quantum number and is integer. In terms of the new variable $x=\cos \theta$, we obtain the following equation for the function $\Theta(\theta) \equiv \Theta(x)$ :

$$
\left[\partial_{x}^{2}+\frac{\tilde{\tau}(x)}{\sigma(x)} \partial_{x}+\frac{\tilde{\sigma}(x)}{\sigma^{2}(x)}\right] \Theta(x)=0,
$$


where $\sigma=1-x^{2}, \tilde{\tau}=-2 x, \tilde{\sigma}=-c_{2} x^{2}-c_{1} x+c_{0}$,

$$
\begin{aligned}
& c_{2}=g+M_{0} \alpha, \\
& c_{1}=M_{0} \beta, \\
& c_{0}=g-m^{2}-M_{0} \gamma .
\end{aligned}
$$

The orthonormalized solutions of equation (58) were found in [36]. We give their explicit form

$$
\begin{array}{r}
\Theta_{k m}(\theta)=c_{k m}\left(\sin ^{2} \frac{\theta}{2}\right)^{-A_{1}}\left(\cos ^{2} \frac{\theta}{2}\right)^{-A_{2}} P_{k}^{\left(-2 A_{1},-2 A_{2}\right)}(\cos \theta), \\
k=0,1,2, \cdots .
\end{array}
$$

Parameters $A_{1,2}<0$ are defined by expressions

$$
A_{1,2}=-\frac{1}{2} \sqrt{m^{2}+M_{0}(\alpha+\gamma \pm \beta)}
$$

Because the $M_{0}=2 M(1+\varepsilon) / \hbar^{2}$, then these parameters, in contrary to the results of [36], depend on energy. A function $P_{k}^{(\alpha, \beta)}(x)$, which is the Jacobi polynomials, satisfies the following orthogonality condition [44].

$$
\int_{-1}^{1}(1-x)^{\alpha}(1+x)^{\beta} P_{k}^{(\alpha, \beta)}(x) P_{k^{\prime}}^{(\alpha, \beta)}(x) d x=d_{k}^{2} \delta_{k k^{\prime}}
$$

where the square of the norm of the polynomials is

$$
d_{k}^{2}=\frac{2^{\alpha+\beta+1} \Gamma(k+\alpha+1) \Gamma(k+\beta+1)}{(2 k+\alpha+\beta+1) k ! \Gamma(k+\alpha+\beta+1)}, \quad \alpha>-1, \beta>-1 .
$$

It follows from condition (62) that the angular wave functions (60) for distinct values of $k$ satisfy orthonormal condition

$$
\int_{-1}^{1} \Theta_{k m}(x) \Theta_{k^{\prime} m}(x) d x=\delta_{k k^{\prime}}
$$

Here, the normalization constant $c_{k m}$ is equal to

$$
c_{k m}=\sqrt{\frac{\left(k-A_{1}-A_{2}+1 / 2\right) k ! \Gamma\left(k-2 A_{1}-2 A_{2}+1\right)}{\Gamma\left(k-2 A_{1}+1\right) \Gamma\left(k-2 A_{2}+1\right)}} .
$$

As shown in Ref. [36], separation constant $g$ depends on $k$ and $m$ in this form

$$
\begin{aligned}
g \equiv & g_{k m}(\varepsilon)=k\left[k+1+\sqrt{m^{2}+M_{0}(\alpha+\gamma+\beta)}\right. \\
& \left.+\sqrt{m^{2}+M_{0}(\alpha+\gamma-\beta)}\right]+\frac{1}{2}\left[\sqrt{m^{2}+M_{0}(\alpha+\gamma+\beta)}\right. \\
& \left.+\sqrt{m^{2}+M_{0}(\alpha+\gamma-\beta)}\right] \\
& +\frac{1}{2} \sqrt{\left[m^{2}+M_{0}(\alpha+\gamma+\beta)\right]\left[m^{2}+M_{0}(\alpha+\gamma-\beta)\right]} \\
& +\frac{1}{2}\left[m^{2}+M_{0}(\gamma-\alpha)\right] .
\end{aligned}
$$

Thus, the exact discrete energy eigenvalues of the KFG equation for our system are defined as follows:

$$
\left(n+\frac{1}{2}+\sqrt{\frac{1}{4}+g_{k m}(\varepsilon)}\right) \sqrt{1-\varepsilon}=\alpha_{0} \sqrt{1+\varepsilon}
$$

This equation implies that the calculation of the energy levels becomes very complicated. We consider two special cases of formulas (21) and (60).

(1) For $f(\theta, \phi)=0$ in equation (6), the potential we used becomes Coulomb potential. In this case, $g=l(l+1), \quad v=l+1$, and we will have $A_{1}=A_{2}=-$ $|m| / 2, l=k+|m|$

$$
\begin{aligned}
\Theta_{l m}(\theta)= & (-1)^{(m-|m|) / 2} \frac{1}{2^{|m|} l !} \\
& \cdot \sqrt{\frac{2 l+1}{2}(l-|m|) !(l+|m|) !(\sin \theta)^{|m|} P_{l-|m|}^{(|m|,|m|)}(\cos \theta) .}
\end{aligned}
$$

Letting $\alpha=\beta=\gamma=0$ in (67) and solving the corresponding energy equation, we obtain for the Coulomb potential

$$
\frac{E_{n l}}{M c^{2}}=\varepsilon_{n l}=\left[1-\frac{2 \alpha_{0}^{2}}{\alpha_{0}^{2}+\hbar^{2} c^{2}(n+l+1)^{2}}\right] .
$$

At the nonrelativistic limit, we get the following formula

$$
\lim _{c \rightarrow \infty}\left(E_{n}-M c^{2}\right)=E_{N n}=-\frac{2 M \alpha_{E}^{2}}{\hbar^{2}(n+l+1)^{2}} .
$$

The corresponding radial wave functions have the form

$$
R_{n l}(\rho)=\sqrt{\frac{n !\left(1-\varepsilon_{n}^{2}\right)^{l+3 / 2}}{(n+l+1) \Gamma(n+2 l+2)}}(2 \rho)^{l+1} e^{-\sqrt{1-\varepsilon_{n}^{2}} \rho} L_{n}^{2 l+1}\left(2 \sqrt{1-\varepsilon_{n}^{2}} \rho\right) .
$$


Thanks to the connecting formula [41] between the Jacobi polynomials $P_{n}^{(\alpha, \alpha)}(x)$ and the Gegenbauer polynomials $C_{n}^{\lambda}(x)$

$$
\begin{aligned}
\left(\lambda+\frac{1}{2}\right)_{n} C_{n}^{\lambda}(x) & =(2 \lambda)_{n} P_{n}^{(\lambda-(1 / 2), \lambda-(1 / 2))}(x), \\
(\lambda)_{n} & =\frac{\Gamma(n+\lambda)}{\Gamma(\lambda)}
\end{aligned}
$$

we can express the functions $\Theta_{l m}(\theta)$ (68) through the polynomials $C_{n}^{\lambda}(x)$, i.e.,

$$
\begin{aligned}
\Theta_{l m}(\theta)= & (-1)^{(m-|m|) / 2} 2^{|m|} \Gamma\left(|m|+\frac{1}{2}\right) \\
& \cdot \sqrt{\frac{2 l+1}{2 \pi} \frac{(l-|m|) !}{(l+|m|) !}}(\sin \theta)^{|m|} C_{l-|m|}^{|m|+1 / 2}(\cos \theta) .
\end{aligned}
$$

On the other hand, there is the connecting formula [41]

$$
P_{l}^{|m|}(\cos \theta)=\frac{(-2)^{|m|}}{\sqrt{\pi}} \Gamma\left(|m|+\frac{1}{2}\right)(\sin \theta)^{|m|} C_{l-|m|}^{|m|+1 / 2}(\cos \theta)
$$

between the Gegenbauer polynomials $C_{n}^{\lambda}(\cos \theta)$ and the associated Legendre functions $P_{l}^{|m|}(\cos \theta)$. We have instead of the formula (73) [1]

$$
\Theta_{l m}(\theta)=(-1)^{(m+|m|) / 2} \sqrt{\frac{2 l+1}{2} \frac{(l-|m|) !}{(l+|m|) !}} l_{l}^{|m|}(\cos \theta) .
$$

Hence, the total wave functions take the form

$$
\begin{aligned}
\psi_{n k m}(r, \theta, \phi)= & C_{n}\left(2 \sqrt{1-\varepsilon_{n}^{2}} \rho\right)^{v} e^{-\sqrt{1-\varepsilon_{n}^{2}} \rho} L_{n}^{2 v-1}\left(2 \sqrt{1-\varepsilon_{n}^{2} \rho}\right) \\
& \cdot\left(\sin ^{2} \frac{\theta}{2}\right)^{-A_{1}}\left(\cos ^{2} \frac{\theta}{2}\right)^{-A_{2}} P_{k}^{\left(-2 A_{1},-2 A_{2}\right)}(\cos \theta) e^{i m \phi}
\end{aligned}
$$

in the case of a discrete spectrum, and

$$
\begin{aligned}
\psi_{k m}(r, \theta, \phi)= & C \rho^{v} e^{-i \tau \rho} F\left(v+2 i \sqrt{\frac{\varepsilon+1}{\varepsilon-1}} \alpha_{0}, 2 v ; 2 i \sqrt{\varepsilon^{2}-1} \rho\right) \\
& \cdot\left(\sin ^{2} \frac{\theta}{2}\right)^{-A_{1}}\left(\cos ^{2} \frac{\theta}{2}\right)^{-A_{2}} P_{k}^{\left(-2 A_{1},-2 A_{2}\right)}(\cos \theta) e^{i m \phi}
\end{aligned}
$$

in the case of a continuous spectrum.

(2) At $\gamma=\beta=0$, equation (60) coincides with equation (16) of Ref. [34]. (The relationship between our parameter $v$ and parameter $L$ of Ref. [34] is as follows $v=L+1$.)

\section{The Nonrelativistic Limit}

Let us now show that the energy spectrum and the radial wave functions considered in previous sections in the nonrelativistic limit $c \longrightarrow \infty$ pass into the energy spectrum and the radial wave functions of the hydrogen-like atoms, respectively. We have

$$
\begin{gathered}
\lim _{c \rightarrow \infty} A_{1,2}=\left.A_{1,2}\right|_{\varepsilon=1}=A_{N 1,2}, \\
\lim _{c \rightarrow \infty} v=\left.v\right|_{\varepsilon=1}=v_{N},
\end{gathered}
$$$$
\lim _{c \rightarrow \infty} 2 \sqrt{1-\varepsilon_{n}^{2}} \rho=\frac{2 \rho_{N}}{n+v_{N}},
$$$$
\rho_{N}=\frac{2 M \alpha_{E}}{\hbar^{2}} r
$$

$\lim _{c \rightarrow \infty}\left(E_{n}-M c^{2}\right)=E_{N n}=-\frac{2 M \alpha_{E}^{2}}{\hbar^{2}\left(n+v_{N}\right)^{2}}$,

$\lim _{c \rightarrow \infty}\left(E_{\lambda}-M c^{2}\right)=E_{N \lambda}=\frac{2 M \alpha_{E}^{2}}{\hbar^{2} \lambda^{2}}$

$$
\begin{aligned}
\lim _{c \rightarrow \infty} \sqrt{\frac{\varepsilon+1}{\varepsilon-1}} \alpha_{0}=\sqrt{\frac{2 M \alpha_{E}^{2}}{\hbar^{2} E_{N \lambda}^{2}}} \equiv \frac{1}{k}, \\
\lim _{c \rightarrow \infty} C_{n}=C_{N n}=\sqrt{\frac{n !}{2\left(n+v_{N}\right) \Gamma\left(n+2 v_{N}\right)}},
\end{aligned}
$$

$\lim _{c \rightarrow \infty} 2 i \sqrt{\varepsilon^{2}-1} \rho=2 i k \rho_{N}$.

Accounting equation (78), we obtain that the nonrelativistic limits of wave functions are equal to

$$
\begin{aligned}
\lim _{c \rightarrow \infty} R_{n}(\rho) & =R_{N n}\left(\rho_{N}\right) \\
& =C_{N n}\left(\frac{2 \rho_{N}}{n+v_{N}}\right)^{v_{N}} e^{-\left(\rho_{N} /\left(n+v_{N}\right)\right)} L_{n}^{2 v_{N}-1}\left(\frac{2 \rho_{N}}{n+v_{N}}\right), \\
\lim _{c \rightarrow \infty} R(\rho) & =R_{N}\left(\rho_{N}\right) \\
& =C_{N}\left(\rho_{N}\right)^{v_{N}} e^{-i k \rho_{N}} F\left(v_{N}+\frac{i}{k}, 2 v_{N} ; 2 i k \rho_{N}\right) .
\end{aligned}
$$

We now give the nonrelativistic limit of operators (46)

$$
\begin{aligned}
& \Gamma_{0}^{N}=\lim _{c \rightarrow \infty} \Gamma_{0}^{\prime}=\frac{1}{2}\left(-\xi \partial_{\xi}^{2}+\frac{g_{N}}{\xi}+\xi\right), \\
& \Gamma_{4}^{N}=\lim _{c \rightarrow \infty} \Gamma_{4}^{\prime}=\frac{1}{2}\left(-\xi \partial_{\xi}^{2}+\frac{g_{N}}{\xi}-\xi\right), \\
& T^{N}=\lim _{c \rightarrow \infty} T^{\prime}=T=-i \xi \partial_{\xi} .
\end{aligned}
$$

\section{Conclusion}

In this paper, the exact solutions of the KFG equation are obtained for equal scalar and vector Coulomb plus ring- 
shaped potentials. The KFG equation coincides in form with the Schrödinger equation for this potential. We solved the wave equation in the standard way by the separation of variables. For the radial wave equation, we constructed a dynamical symmetry group $S U(1,1)$, which in turn allowed us to find the corresponding discrete and continuous energy spectrum of the system by purely algebraically. We also found an explicit form of the wave functions corresponding to the discrete and continuous energy spectra. They were expressed via associated Laguerre polynomials or by confluent hypergeometric functions.

It is found that the radial wave functions, corresponding to both discrete and continuous energy eigenvalues, have the correct nonrelativistic limits. Angular wave functions $\Theta_{k m}(\theta)$ are expressed in terms of Jacobi polynomials, and they are a generalization of the result of [16] to the relativistic case. Therefore, in the nonrelativistic limit, wave functions $\Theta_{k m}(\theta)$ reproduce the results in Ref. [16].

It was also shown that the radial part of the equation of the motion possesses $S U(1,1)$ dynamical symmetry groups. In this line, our work may enable to provide a promising avenue in many branches of physics.

In the literature, there are special cases of our results. For instance, (1) at $f(\theta, \phi)=0$ from our results, one obtains the known results of the corresponding problem (see for example [1]); (2) at $\beta=\gamma=0$ from formulas (21) and (24) for the discrete energy spectrum and radial wave functions, the corresponding formulas of Ref. [34] are obtained; and (3) at $\beta=\alpha=0$, we obtain from our formulas (21) and (22) the corresponding formulas (11) and (15) of Ref. [45].

The main results of this paper are the explicit and closed form expressions for the energy spectrum and corresponding wave functions. We have also shown the radial part of the equation of the motion which possesses $S U(1,1)$ dynamical symmetry groups.

\section{Data Availability}

No data were used to support this study.

\section{Conflicts of Interest}

The authors declare that they have no conflicts of interest.

\section{References}

[1] W. Greiner, Relativistic Quantum Mechanics, Springer, Berlin, 3rd edition, 2000.

[2] V. G. Bagrov and D. M. Gitman, Exact Solutions of Relativistic Wave Equations, Kluwer Academic Publishers, Dordrecht, 1990.

[3] H. Feshbach and F. Villars, "Elementary relativistic wave mechanics of spin 0 and spin 1/2 particles," Reviews of Modern Physics, vol. 30, no. 1, pp. 24-45, 1958.

[4] V. G. Kadyshevsky, R. M. Mir-Kasimov, and N. B. Skachkov, "Three-dimensional formulation of the relativistic twoparticle problem," Physics of Elementary Particles and Atomic Nuclei, vol. 2, p. 635, 1971.

[5] E. D. Kagramanov, R. M. Mir-Kasimov, and S. M. Nagiyev, "The covariant linear oscillator and generalized realization of the dynamical SU(1,1) symmetry algebra," Journal of Mathematical Physics, vol. 31, no. 7, pp. 1733-1738, 1990.

[6] S. M. Nagiyev, "On a hidden symmetry of a relativistic Coulomb problem in the quasipotential approach," Journal of Physics A: Mathematical and General, vol. 21, no. 11, pp. 2559-2564, 1988.

[7] A. Kratzer, "Die ultraroten Rotationsspektren der Halogenwasserstoffe," Zeitschrift für Physik, vol. 3, no. 5, pp. 289307, 1920.

[8] P. M. Morse, "Diatomic Molecules According to the Wave Mechanics. II. Vibrational Levels," Physical Review, vol. 34, no. 1, pp. 57-64, 1929.

[9] C. Eckart, "The Penetration of a Potential Barrier by Electrons," Physical Review, vol. 35, no. 11, pp. 1303-1309, 1930.

[10] M. F. Manning and N. Rosen, "A potential function for the vibrations of diatomic molecules," Physical Review, vol. 44, no. 11, pp. 951-954, 1933.

[11] G. Pöschl and E. Teller, "Bemerkungen zur Quantenmechanik des anharmonischen Oszillators," Zeitschrift für Physik, vol. 83, no. 3-4, pp. 143-151, 1933.

[12] L. Hulthèn, "Uber die Eigenlösungen der Schrödinger chung des Deutrons," Arkiv för Matematik, Astronomi och Fysik, vol. 29, no. 1, p. 1, 1942.

[13] R. D. Woods and D. S. Saxon, "Diffuse surface optical model for nucleon-nuclei scattering," Physical Review, vol. 95, no. 2, pp. 577-578, 1954.

[14] A. A. Makarov, J. A. Smorodinsky, K. Valiev, and P. Winternitz, "A systematic search for nonrelativistic systems with dynamical symmetries," Il Nuovo Cimento A Series 10, vol. 52, no. 4, pp. 1061-1084, 1967.

[15] H. Hartmann, "Die Bewegung eines Körpers in einem ringförmigen Potentialfeld," Theoretica Chimica Acta, vol. 24, no. 2-3, pp. 201-206, 1972.

[16] A. Hautot, "Exact motion in noncentral electric fields," Journal of Mathematical Physics, vol. 14, no. 10, pp. 1320-1327, 1973.

[17] F. Cooper, A. Khare, and U. Sukhatme, Supersymmetry in Quantum Mechanics, World Scientific, 2001.

[18] F. Cooper, A. Khare, and U. Sukhatme, "Supersymmetry and quantum mechanics," Physics Reports, vol. 251, no. 5-6, pp. 267-385, 1995.

[19] S.-H. Dong, Factorization Method in Quantum Mechanics, Springer, Dordrecht, 2007.

[20] J. L. Schiff, The Laplace Transform: Theory and Applications, Springer, New York, NY, USA, 1999.

[21] J. M. Cai, P. Y. Cai, and A. Inomata, "Path-integral treatment of the Hulthén potential," Physical Review A, vol. 34, no. 6, pp. 4621-4628, 1986.

[22] A. Z. Tang and F. T. Chan, "Shifted $1 / \mathrm{N}$ expansion for the Hulthén potential," Physical Review A, vol. 35, no. 2, pp. 911-914, 1987.

[23] B. Roy and R. Roychoudhury, "The shifted 1/N expansion and the energy eigenvalues of the Hulthen potential for 1 not $=0$," Journal of Physics A: Mathematical and General, vol. 20, no. 10, pp. 3051-3055, 1987.

[24] F. Dominguez-Adame, "Bound states of the Klein-Gordon equation with vector and scalar Hulthén-type potentials," Physics Letters A, vol. 136, no. 4-5, pp. 175-177, 1989.

[25] G. Chen, Z. D. Chen, and Z. M. Lou, "Exact bound state solutions of the s-wave Klein-Gordon equation with the 
generalized Hulthén potential," Physics Letters A, vol. 331, no. 6, pp. 374-377, 2004.

[26] B. Talukdar, A. Yunus, and M. R. Amin, "Continuum states of the Klein-Gordon equation for vector and scalar interactions," Physics Letters A, vol. 141, no. 7, pp. 326-330, 1989.

[27] L. Chetouani, L. Guechi, A. Lecheheb, T. F. Hammann, and A. Messouber, "Path integral for Klein-Gordon particle in vector plus scalar Hulthén-type potentials," Physica A, vol. 234, no. 1-2, pp. 529-544, 1996.

[28] A. I. Ahmadov, Sh. M. Nagiyev, M. V. Qocayeva, K. Uzun, and V. A. Tarverdiyeva, "Bound state solution of the Klein-FockGordon equation with the Hulthén plus a ring-shaped-like potential within SUSY quantum mechanics," International Journal of Modern Physics A, vol. 33, article 1850203, 2018.

[29] A. I. Ahmadov, M. Demirci, S. M. Aslanova, and M. F. Mustamin, "Arbitrary $l$-state solutions of the Klein-Gordon equation with the Manning-Rosen plus a Class of Yukawa potentials," Physics Letters A, vol. 384, no. 12, article 126372, 2020.

[30] I. B. Okon, O. Popoola, and C. N. Isonguyo, "Approximate solutions of Schrodinger equation with some diatomic molecular interactions using Nikiforov-Uvarov method," Advances in High Energy Physics, vol. 2017, Article ID 9671816, 24 pages, 2017.

[31] Y.-F. Cheng and T.-Q. Dai, "Solution of the Dirac equation for ring-shaped modified Kratzer potential," Communications in Theoretical Physics, vol. 48, no. 3, pp. 431-434, 2007.

[32] C.-Y. Chen, D.-S. Sun, and F.-L. Lu, "Approximate analytical solutions of Klein-Gordon equation with Hulthén potentials for nonzero angular momentum," Physics Letters A, vol. 370, no. 3-4, pp. 219-221, 2007.

[33] W.-C. Qiang, R.-S. Zhou, and Y. Gao, "Any l-state solutions of the Klein-Gordon equation with the generalized Hulthén potential," Physics Letters A, vol. 371, no. 3, pp. 201-204, 2007.

[34] S.-H. Dong and M. Lozada-Cassou, "Exact solutions of the Klein-Gordon equation with scalar and vector ring-shaped potentials," Physica Scripta, vol. 74, no. 2, pp. 285-287, 2006.

[35] G.-F. Wei, Z.-Z. Zhen, and S.-H. Dong, "The relativistic bound and scattering states of the Manning-Rosen potential with an improved new approximate scheme to the centrifugal term," Central European Journal of Physics, vol. 7, no. 1, p. 175, 2009.

[36] Sh. M. Nagiyev and A. I. Ahmadov, "Exact solution of the relativistic finite-difference equation for the Coulomb plus a ring-shaped-like potential," International Journal of Modern Physics A, vol. 34, no. 17, article 1950089, 2019.

[37] A. O. Barut and R. Raczka, Theory of Group Representations and Applications, Polish Scientific Publishers, Warszawa, 1977.

[38] C. C. Gerry, "Dynamical group for a ring potential," Physics Letters A, vol. 118, no. 9, pp. 445-447, 1986.

[39] V. Fack, H. de Meyer, and G. vanden Berghe, "Dynamic-group approach to the $x^{2}+\lambda x^{2} /\left(1+g x^{2}\right)$ potential," Journal of Mathematical Physics, vol. 27, no. 5, pp. 1340-1343, 1986.

[40] P. Matthys and H. de Meyer, "Dynamical-group approach to the Hulthén potential," Physical Review A, vol. 38, no. 3, pp. 1168-1171, 1988.

[41] H. Bateman and A. Erdélyi, Higher Transcendental Functions, vol. 2, McGrow-Hill Book Company, New York, NY, USA, 1953.

[42] B. L. Voronov, D. M. Gitman, A. D. Levin, and R. Ferreira, "Peculiarities of the electron energy spectrum in the Coulomb field of a superheavy nucleus," Theoretical and Mathematical Physics, vol. 187, no. 2, pp. 633-648, 2016.
[43] S. M. Nagiyev, "Dynamical symmetry group of the relativistic Coulomb problem in the quasipotential approach," Theoretical and Mathematical Physics, vol. 80, no. 1, pp. 697-702, 1989.

[44] R. Koekoek, P. A. Lesky, and R. F. Swarttouw, Hypergeometric Orthogonal Polynomials and their Q-Analogies, SpringerVerlag, Berlin, 2010.

[45] W.-C. Qiang, "Bound states of the Klein-Gordon and Dirac equations for potential $V(r)=A \bar{r}^{2}-B \bar{r}^{1}$," Chinese Physics, vol. 12, no. 10, pp. 1054-1057, 2003. 